\title{
Analysis and Calibration of CRF Raman Lidar Cloud Liquid Water Measurements
}

\author{
Dr. David D. Turner, Principal Investigator \\ Space Science and Engineering Center \\ University of Wisconsin - Madison \\ 1225 West Dayton Street \\ Madison, WI 53706 \\ 608-263-1061; dturner@ssec.wisc.edu \\ Dr. David N. Whiteman, Co-Investigator \\ NASA Goddard Space Flight Center \\ Mesoscale Atmospheric Processes Branch \\ Greenbelt, MD 20771 \\ 301-614-6703; david.whiteman@gsfc.nasa.gov \\ Dr. Felicita Russo, Co-Investigator \\ University of Maryland Baltimore County \\ 1000 Hilltop Circle, \\ Baltimore, MD 21250 \\ 301-614-6716; rfeli@umbc.edu
}

DOE Program Manager: Dr. Kirankumar Alapaty

Period of Performance: 1 Oct 2006 - 30 Sep 2007

KEYWORDS:

Raman lidar, liquid water content, cloud droplet effective radius, aerosol indirect effect

OBJECTIVES:

- Calibrate the liquid water content profiles measured by the ARM CRF Raman lidar

- Identify and characterize the error sources in these liquid water content observations

\section{RESEARCH APPROACH:}

The Atmospheric Radiation Measurement (ARM) Raman lidar (RL), located at the Southern Great Plains (SGP) Climate Research Facility (CRF), is a unique state-of-the-art active remote sensor that is able to measure profiles of water vapor, aerosol, and cloud properties at high temporal and vertical resolution throughout the diurnal cycle. In October 2005, the capability of the RL was extended by the addition of a new detection channel that is sensitive to the Raman scattering of liquid water. This new channel permits the system, in theory, to measure profiles of liquid water content (LWC) by the RL. To our knowledge, the ARM RL is the only operation lidar with this capability.

The liquid water Raman backscattering cross-section is a relatively weak and spectrally broad feature, which requires that the bandpass of the interference filter used to isolate this signal be several $(\sim 6)$ nanometers wide; by contrast, the bandpass of the interference 
filter used to select the water vapor Raman backscatter signal is only $0.4 \mathrm{~nm}$ wide. The wide bandpass required to achieve reasonable signal-to-noise in the liquid water channel essentially eliminates the ability to measure LWC profiles during the daytime in the presence of large solar background, and thus all LWC observations are nighttime only. Additionally, the wide bandpass increases the probability that other undesirable signals, such as fluorescence from aerosols, may contaminate the observation. The liquid water Raman cross-section has a small amount of overlap with the water vapor Raman crosssection, and thus there will be a small amount of 'cross-talk' between the two signals, with water vapor contributing a small amount of signal to the LWC observation. And finally, there is significant uncertainty in the actual strength of the liquid water Raman cross-section in the literature.

The calibrated LWC profiles, together with the coincident cloud backscatter observations also made by the RL, can be used to derive profiles of cloud droplet effective radius. By combining these profiles of effective radius in the lower portion of the cloud with the aerosol extinction measurements made below the cloud by the RL, the first aerosol indirect effect can be investigated using a single instrument, thereby reducing the uncertainty associated with aligning the different sampling periods and fields of view of multiple instruments.

We have applied a "first principles" calibration to the LWC profiles. This approach requires that the relative differences in optical efficiency between the water vapor and liquid water channels be known; this relative difference is easily computed using the efficiency values of the beam splitters and interference filters in the lidar that were provided by the vendors of these components. The first principles approach then transfers the calibration from the water vapor mixing ratio to the LWC using the difference in the optical efficiency and an interpolated value of the liquid water Raman cross section from the literature, and the better established water vapor Raman cross section.

After accounting for all known error sources, the vertical integral of LWC was compared against a similar value retrieved from a co-located ground-based infrared radiometer. The RL and infrared radiometer have significantly different fields of view; thus to compare the two sensors the data were averaged to 5 min intervals where only cloudy samples were included in the average of each. While there is fair scatter in the data $(\mathrm{r}=0.47)$, there is also a clear indication of a positive correlation between the infrared and the RL values. The value of the slope of the regression is 0.49 , which indicates a tendency of the RL measurements to underestimate the total liquid amount with respect to the infrared retrieval. Research continues to investigate the source of the bias, but the most likely candidate is the large uncertainty in the liquid water Raman cross-section as there have been no direct measurements made of this parameter at the lidar's laser wavelength of $355 \mathrm{~nm}$.

The calibrated LWC profile was then used together with the cloud backscatter coefficient profile from the RL to derive profiles of cloud droplet effective radius and cloud droplet number density. These profiles of cloud droplet size together with the aerosol extinction 
observed by the same lidar are used to investigate the aerosol indirect effect in several case studies in August 2006.

Russo F. "An investigation of Raman lidar measurements and their application to the study of the aerosol indirect effect", PhD Thesis (2007).

Russo F., D. N. Whiteman, D. D. Turner, B. B. Demoz, R. M. Hoff, I. Veselovskii, "Measurements of the Aerosol Indirect Effect using a Raman lidar. Part 1: cloud liquid water measurements", manuscript in preparation.

Russo F., D. N. Whiteman, D. D. Turner, B. B. Demoz, R. M. Hoff, I. Veselovskii, "Measurements of the Aerosol Indirect Effect using a Raman lidar. Part 2: the calculation of IE", manuscript in preparation. 